\title{
Two new species of Camallanus (Nematoda: Camallanidae) from freshwater turtles in Queensland, Australia.
}

\begin{abstract}
We describe 2 new species of Camallanus (Nematoda: Camallanidae) from freshwater turtles collected in Queensland, Australia: Camallanus nithoggi n. sp. from Elseya latisternum (Gray) and Camallanus waelhreow n. sp. from Emydura krefftii (Gray), Emydura macquarrii (Gray), and Em. macquarrii dharra Cann. The only Camallanus sp. previously reported from turtles is C. chelonius Baker, 1983 (all other species in the family have been transferred to Serpinema). The 2 new species described here differ from $C$. chelonius in the number of male preanal papillae (7 vs. 6 in C. chelonius), the number of male postanal papillae (5 vs. 4 in C. chelonius), and the number of buccal capsule ridges. Additionally, we removed the tissues overlying the buccal capsule and used scanning electron micrographs (SEM) to show that the peribuccal shields extend laterally from the buccal capsule, the basal ring is separated from the buccal capsule by a narrow isthmus, and there is a buttress along the lateral margin of the buccal capsule that has not previously been observed in species of Camallanus.
\end{abstract}

Keyword: Camallanus; Australia; Turtles. 